\title{
Structure and function of vascular endothelial growth factor and its receptor system
}

\author{
Seong Ah Park ${ }^{1}$, Mi Suk Jeong ${ }^{1}$, Ki-Tae Ha ${ }^{2}$ E Se Bok Jang ${ }^{1, *}$ \\ ${ }^{1}$ Department of Molecular Biology, College of Natural Sciences, Pusan National University, Busan 46241, ${ }^{2}$ Department of Korean Medical \\ Science, School of Korean Medicine and Korean Medicine Research Centre for Healthy Aging, Pusan National University, Yangsan 50612, \\ Korea
}

\begin{abstract}
Vascular endothelial growth factor and its receptor (VEGF-VEGFR) system play a critical role in the regulation of angiogenesis and lymphangiogenesis in vertebrates. Each of the VEGF has specific receptors, which it activates by binding to the extracellular domain of the receptors, and, thus, regulates the angiogenic balance in the early embryonic and adult stages. However, de-regulation of the VEGF-VEGFR implicates directly in various diseases, particularly cancer. Moreover, tumor growth needs a dedicated blood supply to provide oxygen and other essential nutrients. Tumor metastasis requires blood vessels to carry tumors to distant sites, where they can implant and begin the growth of secondary tumors. Thus, investigation of signaling systems related to the human disease, such as VEGF-VEGFR, will facilitate the development of treatments for such illnesses. [BMB Reports 2018; 51(2): 73-78]
\end{abstract}

\section{INTRODUCTION}

Angiogenesis, the physiological process through which new vessels form from pre-existing vessels, is responsible for most, if not all, blood vessel growth during development $(1,2)$. Various angiogenic proteins, including fibroblast growth factors (FGFs), vascular endothelial growth factors (VEGFs/VEGFRs), angiopoietin/Tie receptors, platelet-derived growth factors (PDGFs/PDGFRs), and EphrinB2/EphB4 (3-8) result in the stimulation of angiogenesis. This process is tightly regulated depending on the balance of pro- and anti-angiogenic factors (9). However, if the angiogenesis is not properly controlled, various diseases are induced. For example, excessive

${ }^{*}$ Corresponding author. Tel: +82-51-510-2523; Fax: +82-51-5812544; E-mail: sbjang@pusan.ac.kr

https://doi.org/10.5483/BMBRep.2018.51.2.233

Received 12 December 2017, Revised 3 January 2018, Accepted 16 January 2018

Keywords: Angiogenesis, Drugs, Signal transduction, Tumor growth and metastasis, Vascular endothelial growth factor (VEGF) angiogenesis can lead to chronic disease states such as tumor growth and metastasis, and several disease, such as ulcers and ischemic heart disease, are the result of insufficient angiogenesis (10). Among the angiogenic proteins, VEGF-VEGFR is a crucial regulator of pathological angiogenesis such as in cancer as well as physiological vasculogenesis and angiogenesis in early embryonic and adult stages (11).

VEGFs bind to the VEGFRs on the cell surface, and stimulate cellular responses by causing the receptors to dimerize and become activated through transphosphorylation (12). When cells are deficient of oxygen, namely in hypoxia, the cell produces hypoxia-inducible factor (HIF), which can stimulate the release of VEGF. Thus, hypoxia may be an essential regulator of VEGF expression. Additionally, several diseases characterized by excess angiogenesis are associated with hypoxia-driven de-regulated VEGF expression $(12,13)$. Several antiangiogenic drugs target the VEGF-VEGFR system, including VEGF-neutralizing antibody (bevacizumab), small molecule kinase inhibitors (sunitinib, sorafenib, and apatinib), and humanized monoclonal antibody targeting the extracellular domain of the VEGFR (ramucirumab). However, the resistance mechanisms of cancer and the side effects of drugs limit the use of these drugs in chemotherapy (14). Consequently, a more detailed investigation focused on the pathological angiogenesis, as a therapeutic target, is required for the development of safe and continuously available drugs.

In this review, we describe the structural and functional information regarding the VEGF-VEGFR system to increase understanding of angiogenesis in physiological and pathological processes.

\section{STRUCTURE AND BIOCHEMICAL PROPERTIES OF VEGFRs WITH ITS LIGANDS}

Genes encoding novel tyrosine kinase receptors were isolated in the early 1990s, and the tyrosine kinase receptors that positively and negatively regulate the formation of blood and lymph vessels were denoted VEGFRs $(15,16)$. Three genes are encoding three full-length receptors (VEGFR-1, -2, and -3) and one soluble molecule (sVEGFR-1), and most VEGFRs show similar overall structures that comprise of three primary 
domains. VEGFRs are typically composed of an extracellular ligand-binding domain (ECD) with a seven immunoglobulin (Ig)-like domain, a transmembrane domain and a tyrosine kinase domain split by a kinase insert and a carboxy terminus (Fig. 1A) $(11,17)$. The kinase domains of VEGFRs are the most conserved region, with high sequence identities (78-80\%). The VEGF-VEGFR system plays a central role in the regulation of tumor angiogenesis and can be a potential target for anti-angiogenic therapy. There are five VEGF family members (VEGF-A, VEGF-B, VEGF-C, VEGF-D, and placental growth factor) encoded from the mammalian genome $(3,18)$. Moreover, alternative splicing of primary RNA transcripts from the VEGF gene family generates various isoforms. For example, the isoforms of human VEGF-A are labeled as VEGF-A 121, $_{\text {, }}$ VEGF-A $_{145}$, VEGF-A ${ }_{165}$, VEGF-A ${ }_{189}$ and VEGF-A $\mathrm{A}_{206}$, and homodimeric VEGF-B exists as two different transcripts, VEGF-B ${ }_{167}$ and VEGF-B ${ }_{186}$ (19). Among them, VEGF-A (known as VEGF) is one of the most critical factors for blood vessel formation during early embryogenesis (11). VEGF-A binds to Ig domains 2 and 3 localized in the ECD of VEGFR-1 and VEGFR-2 $(20,21)$. Interestingly, the affinity of VEGF-A to

A

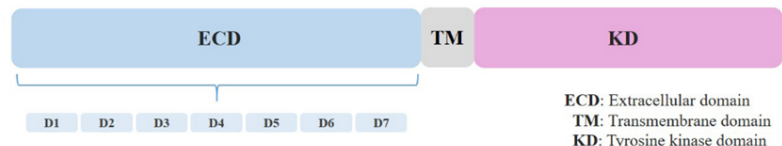

B

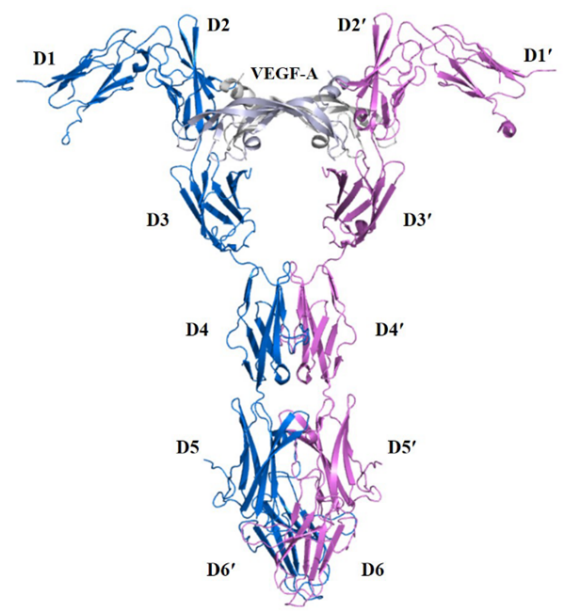

Fig. 1. Structure of the VEGFR-1 extracellular domain in complex with VEGF-A. (A) Schematic representation of the domain organization of VEGFR is shown. (B) Complex crystal structure of VEGFR-1 extracellular domain with VEGF-A (PDB ID: 5T89) is shown. We have shown the structure in a ribbon representation with each chain depicted by a different color. The chains of the VEGF-A homodimer are shown in light blue and gray, and the VEGFR-1 D1-D6 chains in deep blue and magenta.
VEGFR-1 is about one order of magnitude higher than that to VEGFR-2, but the tyrosine kinase activity of VEGFR-2 in response to VEGF-A is much higher than that of VEGFR-1 (17, 22). VEGF-B and placenta growth factor (PIGF) bind to VEGFR-1, but their mechanisms that activate the receptor are different (23). Specifically, VEGF-B stimulates Tyr1213 phosphorylation of VEGFR-1, whereas PIGF stimulates Tyr1309 phosphorylation (24). VEGF-C and VEGF-D are specific ligands for VEGFR-3, which plays a critical role in angiogenesis and lymphangiogenesis in adults (Fig. 2) (25).

To date, many structural studies of the VEGF/VEGFR complex based on single-particle electron microscopy, small-angle $\mathrm{X}$-ray scattering, and X-ray crystallography show how the ligand binds to the membrane distal Ig domains. Moreover, studies of other Ig domains of the VEGFR suggest the possibility of receptor-receptor interactions $(19,26-29)$. The first complete and recently reported VEGF/VEGFR ECD complex structure provides insightful information regarding the ligand binding and ligand-induced homotypic interactions of VEGFR (30). The structure of full-length VEGFR-1 ECD in complex with VEGF-A exists as two sets of 1:1 complexes in the asymmetric unit and two receptors linked by the dimeric VEGF-A bound to the Ig domains (Fig. 1B) (30). Unlike previous VEGFR-1 complex structures that contained only Ig domain 2, the recently reported complex structures include the complete ECD of the receptor with VEGF-A that interacts with both Ig domains 2 and 3 of VEGFR-1 (19, 30-32). The results of these studies also suggest that the homotypic receptor-receptor contacts in Ig domains 4-7 increase the binding affinity of VEGFR-1 ECD for VEGF-A based on the finding that the binding affinity is 20 times higher in the

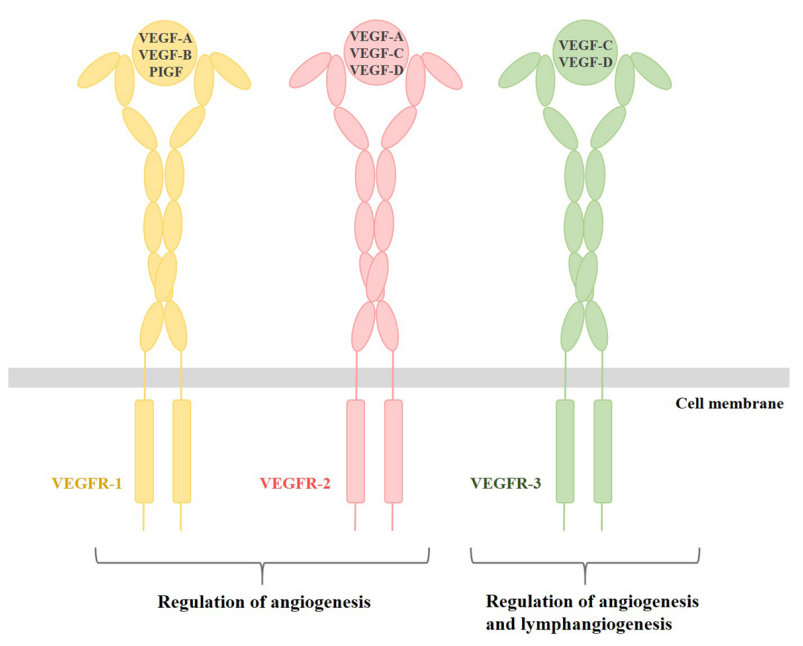

Fig. 2. Schematic illustration of the VEGF-VEGFR system. The VEGF family including VEGF-A, VEGF-B, VEGF-C, VEGF-D and PIGF binds to its specific receptor. We have depicted its ligands in yellow, VEGFR-2 and its ligands in pink, and VEGFR-3 and its ligands in green. 
presence of homotypic interactions (30). Moreover, researchers have conducted many studies targeting the structure-based design of VEGFR-2 inhibitors as therapeutic agents since the crystal structure of the catalytic kinase domain of VEGFR-2 was determined (33-36). The overall fold and catalytic residue positions of the VEGFR-2 kinase domain are similar to those observed in other tyrosine-kinase structures. There are two lobes ( $\mathrm{N}$-lobe and $\mathrm{C}$-lobe), and the catalysis of phosphotransfer takes place in the cleft between the two lobes (34). Despite differences in the kinase activity of VEGFRs in response to its ligands, the available structural information regarding the kinase domains of VEGFR-1 and VEGFR-3 remains sparse. Thus, more detailed investigations based on the molecular structure of the remaining VEGFR kinase domains are required to improve understanding of their catalytic and signal transduction mechanisms.

\section{BIOLOGICAL FUNCTION OF VEGF-VEGFR SYSTEM}

VEGF-VEGFR system is crucial to vascular development and neovascularization in physiological and pathological processes of both embryos and adults, and many studies have investigated anti-VEGF-VEGFR molecules disturbing signal transduction by the VEGF-VEGFR system to improve anti-angiogenic therapy (12). VEGFR-1 is expressed in vascular endothelial cells and non-endothelial cells, including haematopoietic stem cells, macrophages, and monocytes. Fong et al. reported that VEGFR-1 knockout mice died at embryonic day 8.5-9.0 because of overgrowth of endothelial cells and disorganization of blood vessels in the embryo (37). Moreover, to identify how VEGFR-1 negatively regulates angiogenesis during early embryogenesis, mice expressing only the VEGFR-1 extracellular and transmembrane domains were generated. Interestingly, angiogenesis in mice was almost average, indicating that the ECD of VEGFR-1, not the kinase domain, plays a critical role as a suppressor of vascular formation by trapping VEGF-A and thereby preventing VEGFR-2 activation (38). Autophosphorylation on tyrosine residues of VEGFR-1 and coupling to intracellular signal transducers can trigger weak signals for growth and survival of endothelial cells and pericytes, as well as for cell migration of macrophages (17). Phospholipase C $(\mathrm{PLC} \gamma)$ involved in the mitogen-activated protein kinase (MAPK) pathway adheres to the phosphorylated Tyr1169 of VEGFR-1 for regulation of endothelial cell proliferation (39, 40). The p85 subunit of phosphoinositide 3-kinase (PI3K) has also been reported to bind to the activated and phosphorylated VEGFR-1 (41).

The VEGFR-2 expression is detected in not only vascular endothelial and lymphatic endothelial cells, but also megakaryocytes and haematopoietic stem cells (42). In VEGFR-2 knockout mice, there were defects in vasculogenesis and haematopoietic development, resulting in death at embryonic stage 8.5-9.0 (43). These results show that VEGFR-2 acts as a positive signal transducer in growth and differentiation of endothelial cells. Consequently, these findings indicate that VEGFR-1 and VEGFR-2 collaborate in the regulation of vascular formation as a negative and positive regulator, respectively (17). Among the autophosphorylated tyrosine residues in VEGFR-2, phosphorylated Tyr1175 leads to binding of PLC $\gamma$, which stimulates the MAPK pathway involved in the regulation of DNA synthesis, and binding of PI3K involved in cell survival $(17,44)$. It has also been reported that Tyr951, another phosphorylated residue in VEGFR-2, leads to adaptation of T cell-specific adapter (TSA), which regulates actin stress fiber organization and migratory responses of endothelial cells by associating with the cytoplasmic tyrosine kinase Src (Fig. 3) (45). VEGFR-3 is primarily expressed in lymphatic endothelial cells, and activation of VEGFR-3 by interaction with VEGF-C results in proliferation, migration, and survival of lymphatic endothelial cells. Additionally, VEGFR-3 plays an essential role in the development of the vascular network and the cardiovascular system during embryonic development $(46,47)$. There are five tyrosine phosphorylation sites in the VEGFR-3 kinase domain, and the receptor mainly mediates activation of the MAPK pathway $(17,48)$.

\section{ANGIOGENESIS AND ANTI-ANGIOGENIC THERAPY IN CANCER}

Uncontrolled cell growth and proliferation cause cancer, one of the most common diseases in humans. There are several biological hallmarks of cancer, including self-sufficiency in growth signals, insensitivity to anti-growth signals, evading

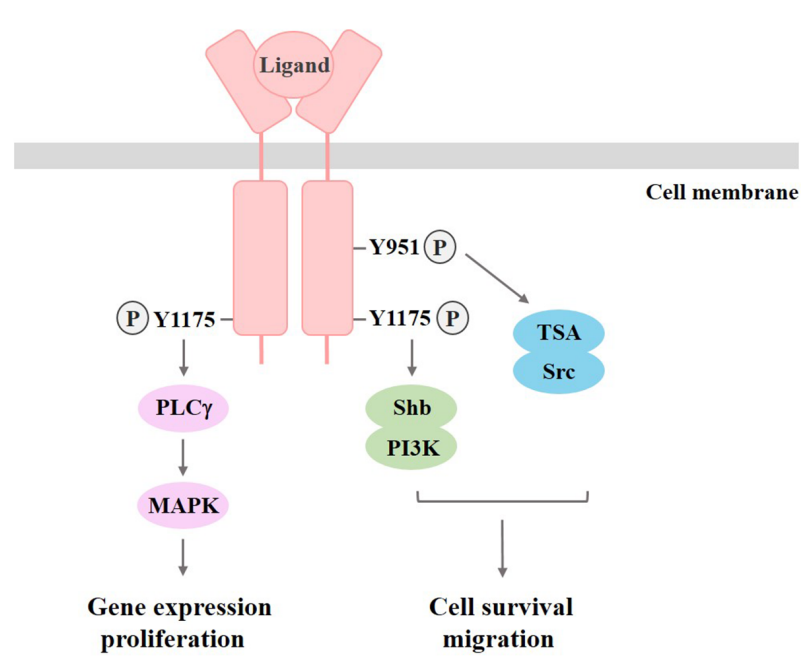

Fig. 3. Signaling pathways activated by VEGFR2. The phosphorylation of tyrosine residues creates docking sites for the recruitment of downstream signaling effectors. Subsequently, signaling cascades activated by VEGFR2 can regulate gene expression, cell proliferation, survival, and migration. 
apoptosis, limitless replicative potential, sustained angiogenesis, tissue invasion and metastasis, abnormal metabolic pathways, evading the immune system, and genome instability (49). Blood vessel growth is essential for the growth and metastasis of solid tumors; thus, angiogenesis is considered one of the most critical targets for investigation of tumor therapy (50). The VEGF-VEGFR system is known as a primary regulator of tumor angiogenesis, and inactivation of the system has been reported in a variety of human diseases such as tumor angiogenesis, tumor-dependent ascites formation, metastasis, and inflammatory diseases including rheumatoid arthritis, rheumatoid psoriasis, hyperthyroidism and atherosclerosis $(3,18)$. VEGFR-1 may contribute to pathological angiogenesis by stimulating the activation of endothelial cells and the recruitment of bone marrow progenitor cells $(51,52)$. Additionally, sVEGFR-1 expressed in the trophoblast layer is a splice variant of VEGFR-1, and may play a critical role in the formation of a regulatory barrier against abnormal vascular permeability and abnormal angiogenesis (11). The finding that artificial overexpression of sVEGFR-1 in a pregnant rat model induces hypertension and proteinuria strongly suggests that increased sVEGFR-1 is a crucial causative factor of the preeclampsia symptoms (hypertension and proteinuria) (53). VEGFR-2 has also been directly linked to tumor angiogenesis and blood vessel-dependent metastasis. Specifically, VEGFR-2 is upregulated under the hypoxic stress that occurs during the rapid growth of tumor cells (11). Either dysfunction or increased activation of VEGFR-3 can be involved in human pathological conditions. Inactivation of VEGFR-3 can aggravate congenital lymphedema that results from decreased transport capacity of the lymphatic vessels and features chronic and disabling swelling of tissues $(54,55)$. Another lymphedema caused by filariasis, trauma or infection may be treated with VEGF-C, alleviating the increased activation of VEGFR-3 (17).

The VEGF-VEGFR system has been confirmed to be useful as a target of new drugs to suppress a range of diseases, particularly malignancies. There are several anti-angiogenic compounds including VEGF-neutralizing antibody (bevacizumab) and tyrosine kinase inhibitor (sunitinib and sorafenib), which inhibit growth and metastasis of tumors. When tumors show drug-resistance to standard cytotoxic therapy, anti-angiogenic compounds may be the ideal drugs for treating cancer patients (11). Bevacizumab is a humanized monoclonal antibody targeting VEGF-A that can selectively neutralize VEGF-A, but not other VEGF family members. The FDA approved Bevacizumab in 2004 for the treatment of cancer. However, it was withdrawn in late 2011 because it has no clear efficacy data on overall survival in large-scale phase III clinical researches such as E2100, AVADO and RIBBON-1 clinical trials $(11,56)$. Bevacizumab has some adverse effects that can be life-threatening, including hypertension, proteinuria, rhinorrhagia, thrombosis and bleeding (57). Additionally, certain cancers are resistance to bevacizumab through several mechanisms, such as the enhancement of alternative pro-angiogenic signaling pathways, recruitment of bone marrow-derived pro-angiogenic cells to the tumor, and increasing of pericyte in tumor (58). Sunitinib malate and sorafenib tosylate can selectively target some protein receptors, including VEGFRs, and inhibit their kinase activity. Moreover, they can be widely applied because they cause few adverse reactions (59). In addition, the development of other anti-VEGF-VEGFR drugs such as VEGF-Trap and humanized anti-VEGFR antibodies is consistently ongoing to overcome adverse drug effects. Recent studies suggest that VEGF pathway appears to be useful for prognosis of several cancers patients including breast cancer and is also conducted as the most critical pathway regulating liver and lymph node metastasis of breast cancer (60-62). Therefore, we can use VEGF-VEGFR system as a potential target of new drugs, and more detailed structure-based insightful information regarding the VEGF-VEGFR system is essential to improve the anti-angiogenic therapy for the improved quality of life of cancer patients.

\section{ACKNOWLEDGEMENTS}

This research was financially supported by the 2017 Post-Doc. Development Program of Pusan National University and Basic Science Research Program through the National Research Foundation of Korea (NRF) funded by the Ministry of Education (2017R1A6A3A11028281).

\section{CONFLICTS OF INTEREST}

The authors have no conflicting interests.

\section{REFERENCES}

1. Birbrair A, Zhang T, Wang ZM, Messi ML, Mintz A and Delbono $O$ (2015) Pericytes at the intersection between tissue regeneration and pathology. Clin Sci (Lond) 128, 81-93

2. Birbrair A, Zhang T, Wang ZM et al (2014) Type-2 pericytes participate in normal and tumoral angiogenesis. Am J Physiol Cell Physiol 307, C25-38

3. Alitalo K and Carmeliet P (2002) Molecular mechanisms of lymphangiogenesis in health and disease. Cancer Cell 1, 219-227

4. Davis S, Aldrich TH, Jones PF et al (1996) Isolation of angiopoietin-1, a ligand for the TIE2 receptor, by secretion-trap expression cloning. Cell 87, 1161-1169

5. Ferrara $N$ and Davis-Smyth T (1997) The biology of vascular endothelial growth factor. Endocr Rev 18, 4-25

6. Heldin CH and Westermark B (1999) Mechanism of action and in vivo role of platelet-derived growth factor. Physiol Rev 79, 1283-1316

7. Shibuya M, Ito N and Claesson-Welsh L (1999) Structure and function of vascular endothelial growth factor receptor-1 and -2. Curr Top Microbiol Immunol 237, 59-83 
8. Wang HU, Chen ZF and Anderson DJ (1998) Molecular distinction and angiogenic interaction between embryonic arteries and veins revealed by ephrin-B2 and its receptor Eph-B4. Cell 93, 741-753

9. Smith GA, Fearnley GW, Harrison MA, Tomlinson DC, Wheatcroft SB and Ponnambalam S (2015) Vascular endothelial growth factors: multitasking functionality in metabolism, health and disease. J Inherit Metab Dis 38, 753-763

10. Carmeliet P and Jain RK (2011) Molecular mechanisms and clinical applications of angiogenesis. Nature 473, 298-307

11. Shibuya M (2013) Vascular endothelial growth factor and its receptor system: physiological functions in angiogenesis and pathological roles in various diseases. J Biochem 153, 13-19

12. Cross MJ, Dixelius J, Matsumoto T and Claesson-Welsh L (2003) VEGF-receptor signal transduction. Trends Biochem Sci $28,488-494$

13. Harris AL (2002) Hypoxia-a key regulatory factor in tumour growth. Nat Rev Cancer 2, 38-47

14. Fontanella C, Ongaro E, Bolzonello S, Guardascione M, Fasola G and Aprile G (2014) Clinical advances in the development of novel VEGFR2 inhibitors. Ann Transl Med 2,123

15. de Vries C, Escobedo JA, Ueno H, Houck K, Ferrara N and Williams LT (1992) The fms-like tyrosine kinase, a receptor for vascular endothelial growth factor. Science 255, 989-991

16. Terman BI, Carrion ME, Kovacs E, Rasmussen BA, Eddy RL and Shows TB (1991) Identification of a new endothelial cell growth factor receptor tyrosine kinase. Oncogene 6, 1677-1683

17. Shibuya $M$ and Claesson-Welsh $L$ (2006) Signal transduction by VEGF receptors in regulation of angiogenesis and lymphangiogenesis. Exp Cell Res 312, 549-560

18. Shibuya $M$ (2011) Involvement of Flt-1 (VEGF receptor-1) in cancer and preeclampsia. Proc Jpn Acad Ser B Phys Biol Sci 87, 167-178

19. Iyer S, Darley PI and Acharya KR (2010) Structural insights into the binding of vascular endothelial growth factor-B by VEGFR-1(D2): recognition and specificity. J Biol Chem 285, 23779-23789

20. Davis-Smyth T, Chen H, Park J, Presta LG and Ferrara N (1996) The second immunoglobulin-like domain of the VEGF tyrosine kinase receptor Flt-1 determines ligand binding and may initiate a signal transduction cascade. EMBO J 15, 4919-4927

21. Tanaka K, Yamaguchi S, Sawano A and Shibuya M (1997) Characterization of the extracellular domain in vascular endothelial growth factor receptor-1 (Flt-1 tyrosine kinase). Jpn J Cancer Res 88, 867-876

22. Waltenberger J, Claesson-Welsh L, Siegbahn A, Shibuya $M$ and Heldin $\mathrm{CH}$ (1994) Different signal transduction properties of KDR and Flt1, two receptors for vascular endothelial growth factor. J Biol Chem 269, 26988-26995

23. Roskoski R Jr (2008) VEGF receptor protein-tyrosine kinases: structure and regulation. Biochem Biophys Res Commun 375, 287-291

24. Autiero M, Waltenberger J, Communi D et al (2003) Role of PIGF in the intra- and intermolecular cross talk between the VEGF receptors Flt1 and Flk1. Nat Med 9, 936-943

25. Roskoski R Jr (2007) Vascular endothelial growth factor (VEGF) signaling in tumor progression. Crit Rev Oncol Hematol 62, 179-213

26. Brozzo MS, Bjelic S, Kisko K et al (2012) Thermodynamic and structural description of allosterically regulated VEGFR-2 dimerization. Blood 119, 1781-1788

27. Leppanen VM, Tvorogov D, Kisko K et al (2013) Structural and mechanistic insights into VEGF receptor 3 ligand binding and activation. Proc Natl Acad Sci U S A 110, 12960-12965

28. Ruch C, Skiniotis G, Steinmetz MO, Walz $T$ and Ballmer-Hofer K (2007) Structure of a VEGF-VEGF receptor complex determined by electron microscopy. Nat Struct Mol Biol 14, 249-250

29. Yang Y, Xie P, Opatowsky $Y$ and Schlessinger J (2010) Direct contacts between extracellular membrane-proximal domains are required for VEGF receptor activation and cell signaling. Proc Natl Acad Sci U S A 107, 1906-1911

30. Markovic-Mueller S, Stuttfeld E, Asthana M et al (2017) Structure of the Full-length VEGFR-1 Extracellular Domain in Complex with VEGF-A. Structure 25, 341-352

31. Christinger HW, Fuh G, de Vos AM and Wiesmann C (2004) The crystal structure of placental growth factor in complex with domain 2 of vascular endothelial growth factor receptor-1. J Biol Chem 279, 10382-10388

32. Wiesmann C, Fuh G, Christinger HW, Eigenbrot C, Wells JA and de Vos AM (1997) Crystal structure at 1.7 A resolution of VEGF in complex with domain 2 of the Flt-1 receptor. Cell 91, 695-704

33. McTigue M, Murray BW, Chen JH, Deng YL, Solowiej J and Kania RS (2012) Molecular conformations, interactions, and properties associated with drug efficiency and clinical performance among VEGFR TK inhibitors. Proc Natl Acad Sci U S A 109, 18281-18289

34. McTigue MA, Wickersham JA, Pinko C et al (1999) Crystal structure of the kinase domain of human vascular endothelial growth factor receptor 2: a key enzyme in angiogenesis. Structure 7, 319-330

35. Okamoto K, Ikemori-Kawada M, Jestel A et al (2015) Distinct binding mode of multikinase inhibitor lenvatinib revealed by biochemical characterization. ACS Med Chem Lett 6, 89-94

36. Oguro Y, Miyamoto N, Okada K et al (2010) Design, synthesis, and evaluation of 5-methyl-4-phenoxy-5Hpyrrolo[3,2-d]pyrimidine derivatives: novel VEGFR2 kinase inhibitors binding to inactive kinase conformation. Bioorg Med Chem 18, 7260-7273

37. Fong GH, Rossant J, Gertsenstein $M$ and Breitman ML (1995) Role of the Flt-1 receptor tyrosine kinase in regulating the assembly of vascular endothelium. Nature 376, 66-70

38. Hiratsuka S, Minowa O, Kuno J, Noda T and Shibuya M (1998) Flt-1 lacking the tyrosine kinase domain is sufficient for normal development and angiogenesis in mice. Proc Natl Acad Sci U S A 95, 9349-9354

39. Landgren E, Schiller P, Cao $Y$ and Claesson-Welsh L (1998) Placenta growth factor stimulates MAP kinase and mitogenicity but not phospholipase C-gamma and 
migration of endothelial cells expressing Flt 1. Oncogene 16, 359-367

40. Sawano A, Takahashi T, Yamaguchi S and Shibuya M (1997) The phosphorylated 1169-tyrosine containing region of flt-1 kinase (VEGFR-1) is a major binding site for PLCgamma. Biochem Biophys Res Commun 238, 487-491

41. Cunningham SA, Waxham MN, Arrate PM and Brock TA (1995) Interaction of the Flt-1 tyrosine kinase receptor with the p85 subunit of phosphatidylinositol 3-kinase. Mapping of a novel site involved in binding. J Biol Chem 270, 20254-20257

42. Katoh O, Tauchi H, Kawaishi K, Kimura A and Satow $Y$ (1995) Expression of the vascular endothelial growth factor (VEGF) receptor gene, KDR, in hematopoietic cells and inhibitory effect of VEGF on apoptotic cell death caused by ionizing radiation. Cancer Res 55, 5687-5692

43. Shalaby F, Rossant J, Yamaguchi TP et al (1995) Failure of blood-island formation and vasculogenesis in Flk-1-deficient mice. Nature 376, 62-66

44. Holmqvist K, Cross MJ, Rolny C et al (2004) The adaptor protein shb binds to tyrosine 1175 in vascular endothelial growth factor (VEGF) receptor-2 and regulates VEGF-dependent cellular migration. J Biol Chem 279, 22267-22275

45. Matsumoto T, Bohman S, Dixelius J et al (2005) VEGF receptor-2 Y951 signaling and a role for the adapter molecule TSAd in tumor angiogenesis. EMBO J 24, 2342-2353

46. Karkkainen MJ, Haiko P, Sainio K et al (2004) Vascular endothelial growth factor $C$ is required for sprouting of the first lymphatic vessels from embryonic veins. Nat Immunol 5, 74-80

47. Makinen T, Veikkola T, Mustjoki S et al (2001) Isolated lymphatic endothelial cells transduce growth, survival and migratory signals via the VEGF-C/D receptor VEGFR-3. EMBO J 20, 4762-4773

48. Dixelius J, Makinen T, Wirzenius M et al (2003) Ligand-induced vascular endothelial growth factor receptor-3 (VEGFR-3) heterodimerization with VEGFR-2 in primary lymphatic endothelial cells regulates tyrosine phosphorylation sites. J Biol Chem 278, 40973-40979

49. Hanahan D and Weinberg RA (2011) Hallmarks of cancer: the next generation. Cell 144, 646-674

50. Folkman J (1971) Tumor angiogenesis: therapeutic implications. N Engl J Med 285, 1182-1186
51. Carmeliet P, Moons L, Luttun A et al (2001) Synergism between vascular endothelial growth factor and placental growth factor contributes to angiogenesis and plasma extravasation in pathological conditions. Nat Med 7, 575-583

52. Hattori K, Heissig B, Wu Y et al (2002) Placental growth factor reconstitutes hematopoiesis by recruiting VEGFR1(+) stem cells from bone-marrow microenvironment. Nat Med 8, 841-849

53. Maynard SE, Min JY, Merchan J et al (2003) Excess placental soluble fms-like tyrosine kinase 1 (sFlt1) may contribute to endothelial dysfunction, hypertension, and proteinuria in preeclampsia. J Clin Invest 111, 649-658

54. Irrthum A, Karkkainen MJ, Devriendt K, Alitalo K and Vikkula M (2000) Congenital hereditary lymphedema caused by a mutation that inactivates VEGFR3 tyrosine kinase. Am J Hum Genet 67, 295-301

55. Rockson SG (2001) Lymphedema. Am J Med 110, 288-295

56. Gerber HP and Ferrara N (2005) Pharmacology and pharmacodynamics of bevacizumab as monotherapy or in combination with cytotoxic therapy in preclinical studies. Cancer Res 65, 671-680

57. Quesada AR, Munoz-Chapuli R and Medina MA (2006) Anti-angiogenic drugs: from bench to clinical trials. Med Res Rev 26, 483-530

58. Piao Y, Henry V, Tiao N et al (2017) Targeting intercellular adhesion molecule-1 prolongs survival in mice bearing bevacizumab-resistant glioblastoma. Oncotarget 8, 96970-96983

59. Motzer RJ, Michaelson MD, Redman BG et al (2006) Activity of SU11248, a multitargeted inhibitor of vascular endothelial growth factor receptor and platelet-derived growth factor receptor, in patients with metastatic renal cell carcinoma. J Clin Oncol 24, 16-24

60. Chen X, Zheng Z, Chen L and Zheng H (2017) MAPK, NFkappaB, and VEGF signaling pathways regulate breast cancer liver metastasis. Oncotarget 8, 101452-101460

61. Chen Y, Liu Y, Wang Y et al (2017) Quantification of STAT3 and VEGF expression for molecular diagnosis of lymph node metastasis in breast cancer. Medicine (Baltimore) 96, e8488

62. Schirosi L, De Summa S, Tommasi S et al (2017) VEGF and TWIST1 in a 16-biomarker immunoprofile useful for prognosis of breast cancer patients. Int J Cancer 141, 1901-1911 